\title{
Profilaxis en pacientes con hemofilia que tienen inhibidores de alta respuesta
}

\section{Prophylaxis in hemophilia patients with high responding inhibitors}

La hemofilia es una enfermedad hereditaria ligada al cromosoma $\mathrm{X}$ donde las mujeres son portadoras y los hombres padecen la enfermedad. Las complicaciones más frecuentes son la artropatía y el desarrollo de inhibidores, ambos vistos más frecuentemente en el fenotipo grave. En la última década estudios demuestran que la profilaxis primaria en niños es efectiva en la prevención del daño articular y mejora la calidad de vida tanto en los niños como en los adultos (1).

Aproximadamente el $20-30 \%$ de los individuos con hemofilia A severa desarrollan alo-anticuerpos contra el FVIII en los primeros años de vida, generalmente en los primeros 50 días de exposición neutralizando el efecto coagulante del FVIII. Desde el punto de vista clínico, el manejo de sangrados en pacientes con inhibidores de alta respuesta ( $>5$ unidades Bethesda) se deben realizar con el uso de agentes "bypass" o de puente como son el factor FVIIa recombinante (rFVIIa) y el concentrado de complejo de protrombina activado CCPa (FEIBA), a diferencia de los anticuerpos de baja respuesta $(<5$ unidades Bethesda) que habitualmente responden al FVIII (2). La presencia de anticuerpos generalmente no cambia el sitio o frecuencia de los sangrados pero si hace más difícil su manejo ya que los agentes puente no son $100 \%$ efectivos, aumentando el riesgo de una hemorragia severa.

Dos estudios europeos demostraron una mayor morbilidad ortopédica, mayor ausentismo escolar y laboral, hospitalizaciones más frecuentes, y en general una disminución en la calidad de vida en pacientes con inhibidores de alta respuesta cuando se compararon con pacientes sin inhibidores $(3,4)$.

El mecanismo de acción de los agentes puente (rFVIIa y CCPa) no está muy bien definido pero ambos productos generan trombina en la ausencia de FVIII/FIX a nivel del sitio de sangrado.
FEIBA actúa en su mayor parte a nivel del complejo protrombinasa convirtiendo protrombina a trombina por medio del FXa a nivel de la superficie de fosfolípidos, mientras el rFVIIa activa al FX en las plaquetas activadas restaurando suficiente trombina para generar un coagulo estable $(5,6)$. Ambos productos pueden causar trombosis cuando se administran en dosis altas repetitivas o en forma secuencial $(7,8)$.

Hoy en día el manejo recomendado para la erradicación de los anticuerpos es a través de la tolerancia inmune que consiste en la administración repetitiva y frecuente de FVIII para restaurar una tolerancia inmunológica al FVIII y volver a establecer hemostasis sin la utilización de agentes puente. Existen varios protocolos que se pueden utilizar y en general la respuesta es de 50-80\%. Aquellos pacientes que permanecen con inhibidores bien sean porque no responden a la tolerancia inmune o no tienen acceso a una, están a mayor riesgo de desarrollar articulaciones blanco (target joint), artropatía avanzada y hemorragias que pueden poner en peligro sus vidas (9). Es así como en los años 70 aparecen los primeros reportes utilizando complejos protrombínicos en forma profiláctica disminuyendo el número de sangrados articulares en pacientes con inhibidores. En los últimos 10 años se han publicado tres estudios prospectivos demostrando reducción en sangrados (articulares y no articulares) y mejora en la calidad de vida en pacientes que recibieron profilaxis con agentes puente cuando se comparó con la administración de tratamiento a demanda con los mismos productos (10-12). Esta modalidad de manejo en pacientes con inhibidores no se practica frecuentemente a nivel mundial en parte por el costo de los factores y las características en el sistema de salud específicas de cada país.

Según el informe de la Federación Mundial de Hemofilia en Colombia existe un número
Ver artículo: página 288

Dr. Miguel A. Escobar: MD. Professor of Medicine and Pediatrics, University of Texas Health Science Center and MD Anderson Cancer Center, Department of Hematology. Director Gulf States Hemophilia and Thrombophilia Center. Houston, TX (USA)

Correspondencia. Dr. Miguel A. Escobar. Houston, TX (USA). E-Mail: miguel.escobar@uth.tmc. edu 
significativo de pacientes con inhibidores $(n=85)$, los cuales en su mayoría tienen acceso a factores de la coagulación.

En este volumen, Solano y colaboradores describen un estudio prospectivo en cuatro Centros académicos de atención integral de hemofilia en las ciudades de Bogotá y Bucaramanga. El estudio tenía como finalidad evaluar la eficacia de la profilaxis con concentrado de complejo de protrombina activado (FEIBA) en pacientes con hemofilia A e inhibidores de alta respuesta (13).

Los investigadores reportan ocho pacientes con mediana de edad de 18 años, de los cuales todos excepto uno tenían articulaciones blanco. Todos los pacientes habían tenido sangrados previos al inicio de la profilaxis y solo dos no habían tenido hemartrosis previas. La mediana de tiempo de seguimiento fue de 12 meses con una adherencia al tratamiento mayor de $80 \%$, recibiendo dosis entre 40-75 IU/kg.

Los resultados de este estudio prospectivo fueron bastante significativos. Los ocho pacientes tuvieron una mediana de seis sangrados en el periodo pre-profilaxis comparado con un evento de sangrado en el periodo post-profilaxis con un equivalente a 0.67 (IC95\%: 0.50-0.88) y 0.09 eventos por mes (IC 95\%:0.04-0.19) respectivamente. La tasa anual de sangrados articulares fue de 3.1 en el periodo preprofilaxis y de 1 después de 12 meses de profilaxis con FEIBA. La diferencia entre las medianas fue estadísticamente significativa $(\mathrm{p}=0.02)$. El estudio en general demostró una reducción de $86 \%$ de todos los sangrados y de $68 \%$ para las hemartrosis. En este estudio no hubo evidencia de trombosis.

Este estudio colombiano a pesar de incluir un número pequeño de sujetos, demuestra una reducción significativa en el número total de sangrados y hemartrosis en pacientes con inhibidores que recibieron profilaxis con FEIBA. Resultados similares se vieron en los estudios internacionales ProFEIBA y PROOF donde se utilizaron dosis más altas de FEIBA $(\sim 85 \mathrm{IU} / \mathrm{kg})$ con reducciones del $61 \%$ y $72.5 \%$ respectivamente $(11,12)$.

El manejo de pacientes con inhibidores es complejo y costoso sobre todo para los países en desarrollo, sin embargo protocolos como el descrito por Solano y colaboradores utilizando un agente bypass para prevenir sangrados es una opción viable para mejorar la calidad de vida y posiblemente disminuir la artropatía hemofílica.

\section{Referencias}

1. Manco-Johnson MJ,Abshire TC, Shapiro AD, et al. Prophylaxis versus episodic treatment to prevent joint disease in boys with severe hemophilia. N Engl J Med 2007;357(6):535-44

2. Escobar MA, Key N. Hemophilia A and Hemophilia B, in Williams Hematology. Ninth edition. Beutler E., et al editors. McGraw Hill. 2016.

3. Scalone L, Mantovani LG, Mannucci PM, Gringeri A: COCIS study investigators. Quality of life is associated to the orthopedic status in haemophilia patients with inhibitors. Haemophilia 2006;12(2):154-162.

4. Morfini M, Haya S, Tagariello G, et al. European study on orthopedic status of hemophilia patients with inhibitors. Haemophilia 2007;13:606-612.

5. Turecek PL, Varadi K, Gritsch H, Schwarz HP. FEIBA: mode of action. Haemophilia 2004;10(suppl2):3-9.

6. Hoffman M, Monroe DM III, Roberts HR. Activated factor VII activates factor IX and X on the surface of activated platelets: thoughts on the mechanism of action of high-dose activated FVII. Blood Coagul Fibrinolysis 1998; 9(suppl 1): S61-S65.

7. Aledort LM. Comparative thrombotic event incidence after infusion of recombinant factor VIIa versus factor VIII inhibitor bypass activity. J Thromb Haemost 2004;2(10):1700-08.

8. Schneiderman J, Nugent DJ, Young G. Sequential therapy with activated prothrombin complex concentrate and recombinant factor VIIa in patients with severe haemophilia and inhibitors. Haemophilia 2004;10(4):347-51

9. Leissinger CA,Singleton T, Kruse-Jarres. How I use bypassing therapy for prophylaxis in patients with hemophilia A and inhibitors. BLOOD 2015;126(2):153-9.

10. Konkle BA, Ebbesen LS, Erhardtsen E, et al. Randomized, prospective clinical trial of recombinant factor VIIa for secondary prophylaxis in hemophilia patients with inhibitors. J Thromb Haemost. 2007;5(9):1904-13.

11. Leissinger CA, Gringeri A, Antmen B, et al. Anti-inhibitor coagulant complex prophylaxis in hemophilia with inhibitors. N Engl J Med 2011;365(18):1684-92.

12. Antunes SV, Tangada S, Stasyshyn O, et al. Randomized comparison of prophylaxis and on-demand regimens with FEIBA NF in the treatment of hemophilia A and B with inhibitors. Haemophilia 2014;20(1):65-72.

13. Solano MH, Linares A, Sossa C. Sarmiento I, Casas C Peña A. Profilaxis con CCPa en pacientes con hemofilia A con inhibidores de alta respuesta. Una estrategia alternativa al estándar de tratamiento. Acta Med Colomb 2015; 40: 288-93. 